Proyecciones Journal of Mathematics

Vol. 36, $\mathrm{N}^{\circ}$ 2, pp. 257-282, June 2017.

Universidad Católica del Norte

Antofagasta - Chile

\title{
Stability, Boundedness and periodic solutions to certain second order delay differential equations
}

\author{
A. T. Ademola \\ Obafemi Awolowo University, Nigeria \\ P. O. Arawomo \\ University of Ibadan, Nigeria \\ A. S. Idowu \\ University of Ilorin, Nigeria \\ Received : May 2016. Accepted : January 2017
}

\begin{abstract}
Stability, boundedness and existence of a unique periodic solution to certain second order nonlinear delay differential equations is discussed. By employing Lyapunov's direct (or second) method, a complete Lyapunov functional is constructed and used to establish sufficient conditions, on the nonlinear terms, that guarantee uniform asymptotic stability, uniform ultimate boundedness and existence of a unique periodic solution. Obtained results complement many outstanding recent results in the literature. Finally, examples are given to show the effectiveness of our method and correctness of our results.
\end{abstract}

Subjclass [2000] : 34C25, 34D20, 34D40, 65L06.

Keywords : Second order; Nonlinear differential equation; Uniform stability; Uniform ultimate boundedness; Existence of a unique periodic solutions 


\section{Introduction}

Qualitative behaviour of solutions of various second order differential equations with and without delay have been extensively discussed in the literature and are still receiving attention of authors because of their practical applications. Readers are referred to the books of Burton et al. [5,6], Driver [8], Hale [11], Kolmanovskii and Myshkis [13], Kuag [15], Lakshmikantham et. al. [16], Yoshizawa [30,31,32], which contain general results on the subject matter and the papers of Ademola [1], Ademola et. al. [2,3], Alaba and Ogundare [4], Domoshnitsky [9], Grioryan [10], Jin and Zengrong [12], Kroopnick [14], Ogundare et. al. [17], Ogundare and Afuwape [18], Ogundare and Okecha [19], Tunç [20]-[24], Wang and Zhu [26], Xu [27], Yeniçerioğlu $[28,29]$ and the references cited therein.

In [18] the authors discussed boundedness and stability properties of solutions of

$$
x^{\prime \prime}+f(x) x^{\prime}+g(x)=p\left(t, x, x^{\prime}\right),
$$

where $f, g$ and $p$ are continuous functions in their respective arguments $t, x$ and $x^{\prime}$. In [20] the author discussed boundedness of solutions to

$$
x^{\prime \prime}+c\left(t, x, x^{\prime}\right)+q(t) b(x)=f(t)
$$

where $c, b, q$ and $f$ are continuous functions defined on $\mathbf{R}^{+} \times \mathbf{R}^{2}, \mathbf{R}, \mathbf{R}^{+}$and $\mathbf{R}^{+}$respectively. Recently, in [4] the authors studied the second order non autonomous damped and forced nonlinear ordinary differential equation of the form

$$
\left[a(t) x^{\prime}\right]^{\prime}+b(t) f\left(x, x^{\prime}\right) x^{\prime}+c(t) g(x)=p\left(t, x, x^{\prime}\right),
$$

where the functions $a, b, c, f, g$ and $p$ depend only on the arguments displayed explicitly.

Finally in [1] the author considered stability, boundedness and existence of unique periodic solutions to the following second order ordinary differential equation

$$
\left[\phi(x) x^{\prime}\right]^{\prime}+g\left(t, x, x^{\prime}\right) x^{\prime}+\varphi(t) h(x)=p\left(t, x, x^{\prime}\right)
$$

where $\phi, g, \varphi, h$ and $p$ are continuous functions in their respective arguments on $\mathbf{R}, \mathbf{R}^{+} \times \mathbf{R}^{2}, \mathbf{R}^{+}, \mathbf{R}$ and $\mathbf{R}^{+} \times \mathbf{R}^{2}$ respectively.

Unfortunately, the problem of uniform asymptotic stability (when the function $p=0$ ), uniform boundedness, uniform ultimate boundedness, existence and uniqueness of periodic solutions to second order nonlinear delay 
differential equation (1.1), where the nonlinear functions $g, h$ and $p$ contain variable deviating arguments and the second ordered derivative contains a variable coefficient, is yet to be investigated. The purpose of this paper therefore is to fill this gap. We will consider

$$
\begin{aligned}
& {\left[\phi(x(t)) x^{\prime}(t)\right]^{\prime}+g\left(t, x(t-\tau(t)), x^{\prime}(t-\tau(t))\right) x^{\prime}(t)+h(x(t-\tau(t))) } \\
= & \mathrm{p}\left(\mathrm{t}, \mathrm{x}(\mathrm{t}-\tau(t)), x^{\prime}(t-\tau(t))\right)
\end{aligned}
$$

where $\phi, g, h, p$ and $\tau$ are continuous functions in their respective arguments, (i.e. $\phi, h: \mathbf{R} \rightarrow \mathbf{R}, \tau: \mathbf{R}^{+} \rightarrow \mathbf{R}^{+}$and $g, p: \mathbf{R}^{+} \times \mathbf{R}^{2} \rightarrow \mathbf{R}$ with $\mathbf{R}:=$ $(-\infty, \infty)$ and $\left.\mathbf{R}^{+}:=[0, \infty)\right)$. The primes indicate differentiation with respect to the independent variable $t$. If $x^{\prime}(t)=y(t) \phi^{-1}(x(t)), \phi(x(t)) \neq 0$ for all $t \geq 0$, then equation (1.1) is equivalent to system of first order delay differential equations

$$
\begin{gathered}
x^{\prime}(t)=y(t) \phi^{-1}(x(t)) \\
\mathrm{y}^{\prime}(t)=p\left(t, x(t-\tau(t)), y(t-\tau(t)) \phi^{-1}(x(t-\tau(t)))\right) \\
+\int_{t-\tau(t)}^{t} h^{\prime}(x(s)) y(s) \phi^{-1}(x(s)) d s-h(x) \\
-\mathrm{g}\left(\mathrm{t}, \mathrm{x}(\mathrm{t}-\tau(t)), y(t-\tau(t)) \phi^{-1}(x(t-\tau(t)))\right) y(t) \phi^{-1}(x(t)),
\end{gathered}
$$

where $0 \leq \tau(t) \leq \alpha, \alpha>0$ is a constant to be determined later and the derivatives $h^{\prime}, \phi^{\prime}$ and $\tau^{\prime}$ exist and continuous for all $x$ and $t$. The work is motivated by the recent works in $[1,3,4,20]$. The obtained results are new, in fact according to our observation from relevant literature, this is the first paper on second order delay differential equations where the highest ordered derivatives contains variable coefficient. In Section 2 we discussed the basic mathematical tools that will be used in the sequel. In Section 3 the main results are stated and proved while examples are given in Section 4 . 


\section{Preliminary Results}

Consider the following general nonlinear non-autonomous delay differential equation

$$
\dot{X}=\frac{d X}{d t}=F\left(t, X_{t}\right), \quad X_{t}=X(t+\theta), \quad-r \leq \theta<0, \quad t \geq 0
$$

where $F: \mathbf{R}^{+} \times C_{H} \rightarrow \mathbf{R}^{n}$ is a continuous mapping, $F(t+\omega, \phi)=F(t, \phi)$ for all $\phi \in C$ and for some positive constant $\omega$. We assume that $F$ takes closed bounded sets into bounded sets in $\mathbf{R}^{n} .(C,\|\cdot\|)$ is the Banach space of continuous function $\varphi:[-r, 0] \rightarrow \mathbf{R}^{n}$ with supremum norm, $r>0$; for $H>0$, we define $C_{H} \subset C$ by $C_{H}=\{\varphi \in C:\|\varphi\|<H,\} C_{H}$ is the open $H$-ball in $C, C=C\left([-r, 0], \mathbf{R}^{n}\right)$. We shall state the following basic results:

Lemma 2.1. (See [32] pp 206). Suppose that $F(t, \phi) \in \bar{C}_{0}(\phi)$ and $F(t, \phi)$ is periodic in $t$ of period $\omega, \omega \geq r$, and consequently for any $\alpha>0$ there exists an $L(\alpha)>0$ such that $\phi \in C_{\alpha}$ implies $|F(t, \phi)| \leq L(\alpha)$. Suppose that a continuous Lyapunov functional $V(t, \phi)$ exists, defined on $t \in \mathbf{R}^{+}$, $\phi \in S^{*}, S^{*}$ is the set of $\phi \in C$ such that with $|\phi(0)| \geq H$ ( $H$ may be large) and that $V(t, \phi)$ satisfies the following conditions:

(i) $a(|\phi(0)|) \leq V(t, \phi) \leq b(\|\phi\|)$, where $a(r)$ and $b(r)$ are continuous, increasing and positive for $r \geq H$ and $a(r) \rightarrow \infty$ as $r \rightarrow \infty$;

(ii) $\dot{V}_{(2.1)}(t, \phi) \leq-c(|\phi(0)|)$, where $c(r)$ is continuous and positive for $r \geq H$.

Suppose that there exists an $H_{1}>0, H_{1}>H$, such that

$$
h L\left(\gamma^{*}\right)<H_{1}-H,
$$

where $\gamma^{*}>0$ is a constant which is determined in the following way: By the condition on $V(t, \phi)$ there exist $\alpha>0, \beta>0$ and $\gamma>0$ such that $b\left(H_{1}\right) \leq a(\alpha), b(\alpha) \leq a(\beta)$ and $b(\beta) \leq a(\gamma) . \gamma^{*}$ is defined by $b(\gamma) \leq a\left(\gamma^{*}\right)$. Under the above conditions, there exists a periodic solution of (2.1) of period $\omega$. In particular, the relation (2.2) can always be satisfied if $h$ is sufficiently small.

Lemma 2.2. (See [32] pp 188). Suppose that $F(t, \phi)$ is defined and continuous on $0 \leq t \leq c, \phi \in C_{H}$ and that there exists a continuous Lyapunov functional $V(t, \phi, \varphi)$ defined on $0 \leq t \leq c, \phi, \varphi \in C_{H}$ which satisfy the following conditions: 
(i) $V(t, \phi, \varphi)=0$ if $\phi=\varphi$;

(ii) $V(t, \phi, \varphi)>0$ if $\phi \neq \varphi$;

(iii) for the associated system

$$
\dot{x}(t)=F\left(t, x_{t}\right), \quad \dot{y}(t)=F\left(t, y_{t}\right)
$$

we have $V_{(2.3)}^{\prime}(t, \phi, \varphi) \leq 0$, where for $\|\phi\|=H$ or $\|\varphi\|=H$, we understand that the condition $V_{(2.3)}^{\prime}(t, \phi, \varphi) \leq 0$ is satisfied in the case $V^{\prime}$ can be defined.

Then, for given initial value $\phi \in C_{H_{1}}, H_{1}<H$, there exists a unique solution of $(2.1)$.

Lemma 2.3. (See [32] pp 190). Suppose that a continuous Lyapunov functional $V(t, \phi)$ exists, defined on $t \in \mathbf{R}^{+},\|\phi\|<H, 0<H_{1}<H$ which satisfies the following conditions:

(i) $a(\|\phi\|) \leq V(t, \phi) \leq b(\|\phi\|)$, where $a(r)$ and $b(r)$ are continuous, increasing and positive,

(ii) $\dot{V}_{(2.1)}(t, \phi) \leq-c(\|\phi\|)$, where $c(r)$ is continuous and positive for $r \geq 0$,

then the zero solution of system (2.1) is uniformly asymptotically stable.

Lemma 2.4. (See [5] pp 317). Let $V: \mathbf{R}^{+} \times C \rightarrow \mathbf{R}$ be continuous and locally Lipschitz in $\phi$. If

(i) $W_{0}\left(\left|X_{t}\right|\right) \leq V\left(t, X_{t}\right) \leq W_{1}\left(\left|X_{t}\right|\right)+W_{2}\left(\int_{t-r(t)}^{t} W_{3}\left(X_{t}(s)\right) d s\right)$ and

(ii) $\dot{V}_{(2.1)}\left(t, X_{t}\right) \leq-W_{4}\left(\left|X_{t}\right|\right)+N$, for some $N>0$ where $W_{i}(i=$ $0,1,2,3,4)$ are wedges.

Then $X_{t}$ of system 2.1 is uniformly bounded and uniformly ultimately bounded for bound $M$. 


\section{Main Results}

We will start with the following notations, let $x(t)=x, y(t)=y, \phi(x(t))=$ $\phi(x), g\left(t, x(t-\tau(t)), x^{\prime}(t-\tau(t))\right) x^{\prime}(t)=g(\cdot)$ and $p\left(t, x(t-\tau(t)), x^{\prime}(t-\right.$ $\tau(t)))=p(\cdot)$. Let $\left(x_{t}, y_{t}\right)$ be any solution of the system (1.2), the continuously differentiable functional used in this investigation is $V=V\left(t, x_{t}, y_{t}\right)$ defined as

$$
\begin{aligned}
& 2 V=\left[a^{2}+b \phi(x)(1+b \phi(x))\right] x^{2}+(1+b \phi(x)) y^{2}+2 a x y \\
& +\int_{-\tau(t)}^{0} \int_{t+s}^{t} \lambda y^{2}(\theta) \phi^{-2}(x(\theta)) d \theta d s,
\end{aligned}
$$

where the function $\phi$ is defined in Section $1, a, b, \lambda$ are positive constants and the value of $\lambda$ will be determined later. Next, we state the main results as follows.

Theorem 3.1. Further to the basic assumptions on the functions $\phi, g, h, \tau$ and $p$, suppose that $a, b, \phi_{0}, \phi_{1}, \alpha, \beta, L$ and $M$ are positive constants such that

(i) $\phi_{0} \leq \phi(x) \leq \phi_{1}, \phi^{\prime}(x) \leq 0$ for all $x$;

(ii) $a \leq g(\cdot)$ for all $t \geq 0, x, y, x(t-\tau(t))$ and $y(t-\tau(t))$;

(iii) $b x \leq h(x) \leq L x$ for all $x \neq 0$;

(iv) $\tau(t) \leq \alpha, \tau^{\prime}(t) \leq \beta, 0<\beta<1$ where

$$
\alpha<\min \left\{\frac{b \phi_{0}}{L}, \frac{2 a b \phi_{0}(1-\beta)}{L\left[1+a+b \phi_{1}+2(1-\beta)\left(1+b \phi_{1}\right)\right]}\right\}
$$

(v) $|p(\cdot)| \leq M, 0<M<\infty$ for all $t \geq 0, x, y, x(t-\tau(t))$ and $y(t-\tau(t))$;

then the solution $\left(x_{t}, y_{t}\right)$ of system (1.2) is uniformly bounded and uniformly ultimately bounded.

Remark 1. We observed the following: 
(i) If $\phi(x)=1, g(\cdot)=a, h(t-\tau(t))=b x$ and $p(\cdot)=0$, equation (1.1) reduces to linear constant coefficients differential equation

$$
x^{\prime \prime}+a x^{\prime}+b x=0,
$$

and conditions (i) to (v) of Theorem 3.1 specializes to the corresponding Routh-Hurwitz criteria $a>0$ and $b>0$.

(ii) When $\phi(x)=1, \tau(t)=0$ and $p(\cdot)=f(t)$, equation (1.1) reduces to a special case discussed in [20], thus Theorem 3.1 includes and improves the results in [20].

(iii) Whenever $\phi(x)=\phi(t), g(\cdot)=a(t) f\left(x, x^{\prime}\right), h(x(t-\tau(t)))=g(x)$ and $p(\cdot)=p\left(t, x, x^{\prime}\right)$, equation (1.1) reduces to that discussed in [4], thus Theorem 3.1 improves the boundedness results in [4].

(iv) When $\phi(x)=1, g(\cdot)=f(x), h(x(t-\tau(t)))=g(x)$ and $p(\cdot)=$ $p\left(t, x, x^{\prime}\right)$, equation (1.1) becomes that considered in [18], hence our results extend the results in [18].

(v) If $g(\cdot)=g\left(t, x, x^{\prime}\right), h(x(t-\tau(t)))=\varphi(t) h(x)$ and $p(\cdot)=p\left(t, x, x^{\prime}\right)$, equation (1.1) coincides with (1.1) in [1], thus the results in [1] are contained in this work.

In what follows we will state and prove a result that would be useful in the proof of Theorem 3.1 and the subsequent results.

Lemma 3.2. Under the hypotheses of Theorem 3.1 there exist positive constants $D_{0}=D_{0}\left(a, b, \phi_{0}\right), D_{1}=D_{1}\left(a, b, \phi_{1}\right)$ and $D_{2}=D_{2}\left(\phi_{0}, \lambda\right)$ such that

$D_{0}\left(x^{2}(t)+y^{2}(t)\right) \leq V\left(t, x_{t}, y_{t}\right) \leq D_{1}\left(x^{2}(t)+y^{2}(t)\right)+D_{2} \int_{-\tau(t)}^{0} \int_{t+s}^{t} y^{2}(\theta) d \theta d s$,

for all $t \geq 0, x$ and $y$. Furthermore, there exist positive constants $D_{3}=$ $D_{3}\left(a, b, \alpha, \beta, L, \phi_{1}\right)$ and $D_{4}=D_{4}\left(a, b, \phi_{1}\right)$ such that

$$
V_{(1.2)}^{\prime} \leq-D_{3}\left(x^{2}(t)+y^{2}(t)\right)+D_{4}(|x(t)|+|y(t)|)|p(\cdot)|,
$$

for all $t \geq 0, x$ and $y$. 
Proof. Let $\left(x_{t}, y_{t}\right)$ be any solution of system (1.2), from equation (3.1) for $x=0=y$, we have $V(t, 0,0)=0$ for all $t \geq 0$. Also the functional $V$ defined in (3.1) can be recast in the form

$2 V=b \phi(x)[1+b \phi(x)] x^{2}+b \phi(x) y^{2}+(a x+y)^{2}+\int_{-\tau(t)}^{0} \int_{t+s}^{t} \lambda y^{2}(\theta) \phi^{-2}(x(\theta)) d \theta d s$.

In view of condition (i) of Theorem $3.1, \phi(x) \neq 0$, and the fact that

$$
\int_{-\tau(t)}^{0} \int_{t+s}^{t} \lambda y^{2}(\theta) \phi^{-2}(x(\theta)) d \theta d s \geq 0
$$

for all $t \geq 0, x$ and $y$, there exists a positive constant $\delta_{0}$ such that

$$
V \geq \delta_{0}\left(x^{2}+y^{2}\right)
$$

for all $t \geq 0, x$ and $y$ where

$$
\delta_{0}:=\frac{1}{2} \min \left\{b \phi_{0}\left(1+b \phi_{0}\right)+\min \{1, a\}, b \phi_{0}+\min \{1, a\}\right\} .
$$

In addition, from inequality (3.6) we find that

$$
\begin{gathered}
V\left(t, x_{t}, y_{t}\right)=0 \quad \text { if and only if } x^{2}+y^{2}=0 ; \\
V\left(t, x_{t}, y_{t}\right)>0 \quad \text { if and only if } x^{2}+y^{2} \neq 0 ; \text { and that } \\
V\left(t, x_{t}, y_{t}\right) \rightarrow+\infty \text { as } x^{2}+y^{2} \rightarrow \infty .
\end{gathered}
$$

Moreover, since $\phi_{0} \leq \phi(x) \leq \phi_{1}$ for all $x$ and the fact that the inequality $2 x y \leq x^{2}+y^{2}$ holds, there exist positive constants $\delta_{1}$ and $\delta_{2}$ such that

$$
V \leq \delta_{1}\left(x^{2}+y^{2}\right)+\delta_{2} \int_{-\tau(t)}^{0} \int_{t+s}^{t} y^{2}(\theta) d \theta d s
$$

for all $t \geq 0, x$ and $y$, where

$$
\delta_{1}:=\frac{1}{2} \max \left\{(1+a) a+\left(1+b \phi_{1}\right) b \phi_{1}, 1+a+b \phi_{1}\right\}
$$

and 


$$
\delta_{2}:=\frac{\lambda}{2 \phi_{0}^{2}}
$$

Inequalities (3.6) and (3.10) established the inequality (3.3) with $\delta_{0}, \delta_{1}$ and $\delta_{2}$ equivalent to $D_{0}, D_{1}$ and $D_{2}$ respectively.

Next, the differentiation of the functional $V$ defined by equation (3.1) with respect to the independent variable $t$ along the solution path of system (1.2) after simplification is

$$
\mathrm{V}_{(1.2)}^{\prime}=-\sum_{j=1}^{2} W_{j}+\sum_{j=3}^{4} W_{j}+\tau(t) \phi^{-2}(x) y^{2}+[a x+(1+b \phi(x)) y] p(\cdot)
$$

$$
-\left[1-\tau^{\prime}(t)\right] \lambda \int_{t-\tau(t)}^{t} \phi^{-2}(x(\theta)) y^{2}(\theta) d \theta
$$

where

$$
\begin{aligned}
& \mathrm{W}_{1}:=\frac{a}{2} \frac{h(x)}{x} x^{2}+b g(\cdot) y^{2} \\
& W_{2}:=\frac{a}{4} \frac{h(x)}{x} x^{2}+a[g(\cdot)-a] \phi^{-1}(x) x y+\frac{1}{2} \phi^{-1}(x)[g(\cdot)-a] y^{2} \\
& +\frac{a}{4} \frac{h(x)}{x} x^{2}+(1+b \phi(x))\left[\frac{h(x)}{x}-b\right] x y+\frac{1}{2} \phi^{-1}(x)[g(\cdot)-a] y^{2} \\
& W_{3}:=\frac{1}{2} \phi^{\prime}(x) y\left[b\left(2 b+\phi^{-1}(x)\right) x^{2}+b \phi^{-1}(x) y^{2}\right] ; \text { and } \\
& W_{4}:=[a x+(1+b \phi(x)) y] \int_{t-\tau(t)}^{t} h^{\prime}(x(s)) y(s) \phi^{-1}(x(s)) d s .
\end{aligned}
$$

Now from conditions (ii) and (iii) of Theorem 3.1, we find that

$$
W_{1} \geq \frac{1}{2} a b\left(x^{2}+y^{2}\right)
$$

for all $t \geq 0, x$ and $y$. Using conditions (i), (ii) and (iii) of Theorem 3.1 in $W_{2}$ we have

$$
\begin{gathered}
W_{2} \geq \frac{a}{4}\left[b x^{2}+\frac{4}{\phi_{1}}(g(\cdot)-a) x y+\frac{2}{a \phi_{1}}(g(\cdot)-a) y^{2}\right] \\
+\frac{1}{4}\left[a b x^{2}+4\left(1+b \phi_{0}\right)\left(\frac{h(x)}{x}-b\right) x y+\frac{2}{\phi_{1}}(g(\cdot)-a) y^{2}\right],
\end{gathered}
$$

for all $t \geq 0, x$ and $y$. Employing estimates

$$
\left[\frac{4}{\phi_{1}}(g(\cdot)-a)\right]^{2}<\frac{b}{a \phi_{1}}(g(\cdot)-a) \text { and }\left[4\left(1+b \phi_{0}\right)\left(\frac{h(x)}{x}-b\right)\right]^{2}<\frac{a b}{\phi_{1}}(g(\cdot)-a)
$$


inequality (3.12) becomes

$$
\begin{gathered}
W_{2} \geq \frac{a}{4}\left[\sqrt{b}|x|-\sqrt{2 a^{-1} \phi_{1}^{-1}(g(\cdot)-a)}|y|\right]^{2} \\
+\frac{1}{4}\left[\sqrt{a b}|x|-\sqrt{2 \phi_{1}^{-1}(g(\cdot)-a)}|y|\right]^{2} \geq 0,
\end{gathered}
$$

for all $t \geq 0, x$ and $y$. Moreover, from hypothesis (i) and let $y>0$ it follows from $W_{3}$ that

$$
\left.y\left[b\left(2 b+\phi^{-1}(x)\right) x^{2}+b \phi^{-1}(x)\right) y^{2}\right] \geq\left(2 b+\phi_{1}^{-1}\right) x^{2}+b \phi_{1}^{-1} y^{2} \geq 0
$$

for all $t \geq 0, x$ and $y$. Using inequality (3.13) and $\phi^{\prime}(x) \leq 0$ for all $x$ in $W_{3}$, we obtain

$$
W_{3} \leq 0
$$

for all $t \geq 0, x$ and $y$. Finally, $\phi_{0} \leq \phi(x) \leq \phi_{1}$ for all $x, h^{\prime}(x) \leq L$ for all $x$ and the inequality $x y \leq \frac{1}{2}\left(x^{2}+y^{2}\right)$ imply that

$$
W_{4} \leq \frac{a L}{2 \phi_{0}} \tau(t) x^{2}+\frac{L}{2 \phi_{0}}\left(1+b \phi_{1}\right) \tau(t) y^{2}+\frac{L}{2 \phi_{0}}\left(1+a+b \phi_{1}\right) \int_{t-\tau(t)}^{t} y^{2}(\theta) d \theta,
$$

for all $t \geq 0, x$ and $y$. Inserting estimate $W_{j}(j=1, \cdots, 4)$ in equation (3.11), we obtain

$$
\begin{gathered}
\quad \mathrm{V}_{(1.2)}^{\prime} \leq-\frac{a b}{2}\left(x^{2}+y^{2}\right)-\left[\left[1-\tau^{\prime}(t)\right] \phi_{1}^{-2} \lambda-\frac{L}{2 \phi_{0}}\left(1+a+b \phi_{1}\right)\right] \int_{t-\tau(t)}^{t} y^{2}(\theta) d \theta \\
+\left[\frac{L}{2 \phi_{0}}\left(1+b \phi_{1}\right)+\phi_{1}^{-2} \lambda\right] \tau(t) y^{2}+\frac{a L}{2 \phi_{0}} \tau(t) x^{2}+\left[a|x|+\left(1+b \phi_{1}\right)|y|\right]|p(\cdot)|
\end{gathered}
$$

for all $t \geq 0, x$ and $y$. Furthermore, $\tau(t) \leq \alpha, \tau^{\prime}(t) \leq \beta, \beta \in(0,1)$ and choose

$$
\lambda:=2^{-1}(1-\beta)^{-1} \phi_{0}^{-1} \phi_{1}^{2} L\left(1+a+b \phi_{1}\right)>0
$$


there exist positive constants $\delta_{3}$ and $\delta_{4}$ such that

$$
V_{(1.2)}^{\prime} \leq-\delta_{3}\left(x^{2}+y^{2}\right)+\delta_{4}(|x|+|y|)|p(\cdot)|,
$$

for all $t \geq 0, x$ and $y$ where

$\delta_{3}:=\frac{1}{2} \min \left\{a\left(b-\frac{\alpha L}{\phi_{0}}\right), a b-\frac{L}{\phi_{0}}\left[1+b \phi_{1}+(1-\beta)^{-1}\left(1+a+b \phi_{1}\right)\right] \alpha\right\}$

and

$$
\delta_{4}:=\max \left\{a, \quad 1+b \phi_{1}\right\} .
$$

Inequality (3.16) satisfies the inequality (3.4) with $\delta_{3}$ and $\delta_{4}$ equivalent to $D_{3}$ and $D_{4}$ respectively. This completes the proof of Lemma $3.2 \square$ Next, we will give the prove of Theorem 3.1, using some of the estimates of Lemma 3.2 .

Proof of Theorem 3.1 Let $\left(x_{t}, y_{t}\right)$ be any solution of system (1.2). From inequalities (3.10) and (3.10) hypothesis (i) of Lemma 2.4 holds. Furthermore, using assumption ( $\mathrm{v}$ ) of Theorem 3.1 in estimate (3.16), noting that $|x|<1+x^{2}$, there exist positive constants $\delta_{5}$ and $\delta_{6}$ such that

$$
V_{(1.2)}^{\prime} \leq-\delta_{5}\left(x^{2}+y^{2}\right)+\delta_{6}
$$

where $\delta_{5}:=\delta_{3}-\delta_{4} M$ with $\delta_{4} M$ choosing sufficiently small and $\delta_{6}:=2 \delta_{4} M$. Inequality (3.18) satisfies condition (ii) of Lemma 2.4. Thus by Lemma 2.4 the solution $\left(x_{t}, y_{t}\right)$ of system (1.2) is uniformly bounded and uniformly ultimately bounded. This completes the proof of Theorem 3.1.

Next, if the forcing term $p(\cdot)$ of equation (1.1) is replaced by a function $p_{1}(t)$ where $p_{1}(t)$ is defined on $\mathbf{R}^{+}$, we obtain a special case of equation (1.1) as

$$
\left[\phi(x) x^{\prime}\right]^{\prime}+g(\cdot) x^{\prime}+h(x(t-\tau(t)))=p_{1}(\cdot) .
$$

Equation (3.19) is equivalent to system of first order equations

$$
\begin{gathered}
x^{\prime}=y \phi^{-1}(x), \\
\mathrm{y}^{\prime}=p_{1}(t)+\int_{t-\tau(t)}^{t} h^{\prime}(x(s)) y(s) \phi^{-1}(x(s)) d s-h(x)-g(\cdot) y \phi^{-1}(x),
\end{gathered}
$$


where the functions $g, h$ and $\phi$ are defined in Section 1. We obtain the following result.

Theorem 3.3. If assumptions (i) to (iv) of Theorem 3.1 hold and assumption $(v)$ is replaced by boundedness of the function $p_{1}(t)$, then the solution $\left(x_{t}, y_{t}\right)$ of system (3.20) is uniformly bounded and uniformly ultimately bounded.

Proof. Let $\left(x_{t}, y_{t}\right)$ be any solution of system (3.20), the remaining part of the prove is similar to the proof of Theorem 3.1 hence it is omitted. This completes the proof of Theorem 3.3.

Furthermore, if the function $p(\cdot)$ of equation (1.1) is replaced by $p_{2}\left(t, x, x^{\prime}\right)$ defined on $\mathbf{R}^{+} \times \mathbf{R}^{2}$, we have the following equation

$$
\left[\phi(x) x^{\prime}\right]^{\prime}+g(\cdot) x^{\prime}+h(x(t-\tau(t)))=p_{2}\left(t, x, x^{\prime}\right) .
$$

Equation (3.21) can be written as system of first order differential equations

$$
\begin{gathered}
x^{\prime}=y \phi^{-1}(x), \\
\mathrm{y}^{\prime}=p_{2}\left(t, x, x^{\prime}\right)+\int_{t-\tau(t)}^{t} h^{\prime}(x(s)) y(s) \phi^{-1}(x(s)) d s-h(x)-g(\cdot) y \phi^{-1}(x),
\end{gathered}
$$

and we have following result.

Theorem 3.4. If the forcing term $p_{2}$ of system (3.22) is bounded and assumptions (i) to (iv) of Theorem 3.1 hold, then the solution $\left(x_{t}, y_{t}\right)$ of system (3.22) is uniformly bounded and uniformly ultimately bounded.

Proof. Let $\left(x_{t}, y_{t}\right)$ be any solution of system (3.22), the remaining part of the prove is similar to the proof of Theorem 3.1 hence it is omitted. This completes the proof of Theorem 3.4.

Next, if $p(\cdot)$ of equation 1.1 is zero we have the following special case

$$
\left[\phi(x) x^{\prime}\right]^{\prime}+g(\cdot) x^{\prime}+h(x(t-\tau(t)))=0 .
$$

Equation (3.23) as system of first order differential equations are as follow 


$$
\begin{gathered}
x^{\prime}=y \phi^{-1}(x), \\
\mathrm{y}^{\prime}=\int_{t-\tau(t)}^{t} h^{\prime}(x(s)) y(s) \phi^{-1}(x(s)) d s-h(x)-g(\cdot) y \phi^{-1}(x),
\end{gathered}
$$

and we have following result.

Theorem 3.5. If assumptions (i) to (iv) of Theorem 3.1 hold, then the trivial solution of system (3.24) is uniformly asymptotically stable.

Proof. Let $\left(x_{t}, y_{t}\right)$ be any solution of system (3.24), from equation (3.1) we have $V(t, 0,0)=0$ for all $t \geq 0$. Moreover, the inequalities (3.6) and (3.10) satisfy condition (i) of Lemma 2.3. Also if $p(\cdot)=0$, estimate (3.16) becomes

$$
V_{3.21}^{\prime} \leq-\delta_{3}\left(x^{2}+y^{2}\right)
$$

for all $t \geq 0, x$ and $y$ where $\delta_{3}$ is defined in (3.17). Inequality (3.25) establish assumption (ii) of Lemma 2.3, hence by Lemma 2.3 the trivial solution of system (3.24) is uniformly asymptotically stable. This completes the proof of Theorem 3.5.

Next, we will state and proofs existence and uniqueness results of the solutions of system (1.2).

Theorem 3.6. If assumptions of Theorem 3.1 hold, then there exists a periodic solution of system 1.2 of period $\omega$.

Proof. Let $\left(x_{t}, y_{t}\right)$ be any solution of system (1.2), from inequalities (3.6), (3.10) and estimate (3.9), assumption (i) of Lemma 2.1 hold. Moreover, using hypothesis (v) of Theorem 3.6 and inequality $|x|+|y| \leq$ $2^{1 / 2}\left(x^{2}+y^{2}\right)^{1 / 2}$ in estimate (3.16) there exist positive constants $\delta_{7}$ and $\delta_{8}$ such that

$$
V_{(1.2)}^{\prime} \leq-\delta_{7}\left(x^{2}+y^{2}\right) \leq 0
$$

for all $t \geq 0, x$ and $y$ provided that

$$
\left(x^{2}+y^{2}\right)^{1 / 2} \geq \delta_{8}
$$


where $\delta_{7}:=\frac{1}{2} \delta_{3}$ and $\delta_{8}:=2^{3 / 2} \delta_{3}^{-1} M$. Inequality (3.26) satisfies assumption (ii) of Lemma 2.1, thus by Lemma 2.1 the periodic solution of system (1.2) exists and is of period $\omega$. This completes the proof of Theorem 3.6.

Theorem 3.7. If assumptions of Theorem 3.1 are satisfied, then there exists a unique solution of system (1.2).

Proof. Let $\left(x_{t}, y_{t}\right)$ be any solution of system (1.2), in view of estimates (3.6), (3.7), (3.9) and (3.26), assumptions of Lemma 2.2 hold, thus by Lemma 2.2 solution of system (1.2) is unique. This completes the proof of Theorem 3.7.

\section{Examples}

Example 4.1. Consider the second order delay differential equation

$$
\begin{aligned}
& {\left[\frac{4\left(1+e^{x}\right)}{\left(3+4 e^{x}\right)} x^{\prime}\right]^{\prime}+\left[\frac{2+t^{2}+x^{2}(t-\tau(t))+x^{\prime 2}(t-\tau(t))}{1+t^{2}+x^{2}(t-\tau(t))+x^{\prime 2}(t-\tau(t))}\right] x^{\prime}+\left[\frac{2+x^{2}(t-\tau(t))}{1+x^{2}(t-\tau(t))}\right] \times} \\
& \text { (4.1) } \quad x(t-\tau(t))=\frac{2+t^{2}+\cos [x(t-\tau(t))]+\cos \left[x^{\prime}(t-\tau(t))\right]}{2\left[t^{2}+\cos [x(t-\tau(t))]+\cos \left[x^{\prime}(t-\tau(t))\right]\right]} .
\end{aligned}
$$

Equation (4.1) is equivalent to

$$
\begin{gathered}
x^{\prime}=\frac{\left(3+4 e^{x}\right)}{4\left(1+e^{x}\right)} y \\
y^{\prime}=\frac{2+t^{2}+\cos [x(t-\tau(t))]+\cos \left[y(t-\tau(t)) \phi^{-1}(x(t-\tau(t)))\right]}{2\left[t^{2}+\cos [x(t-\tau(t))]+\cos \left[y(t-\tau(t)) \phi^{-1}(x(t-\tau(t)))\right]\right]} \\
+\int_{t-\tau(t)}^{t} \frac{2+x^{2}(s)+x^{4}(s)}{\left(1+x^{2}(s)\right)^{2}} d s-\frac{x\left(2+x^{2}\right)}{1+x^{2}} \\
-\left[\frac{2+t^{2}+x^{2}(t-\tau(t))+y^{2}(t-\tau(t)) \phi^{-2}(x(t-\tau(t)))}{1+t^{2}+x^{2}(t-\tau(t))+y^{2}(t-\tau(t)) \phi^{-2}(x(t-\tau(t)))}\right] \times \\
y(t-\tau(t)) \phi^{-1}(x(t-\tau(t))) .
\end{gathered}
$$

Comparing systems (1.2) and (4.2) we find that: 
Stability, Boundedness and periodic solutions to certain second... 271

(i) The function

$$
\phi(x)=\frac{4\left(1+e^{x}\right)}{\left(3+4 e^{x}\right)}=1+\frac{1}{3+4 e^{x}} .
$$

Since

$$
0 \leq \frac{1}{3+4 e^{x}} \leq 1
$$

for all $x$, it follows that

$$
1=\phi_{0} \leq \phi(x) \leq \phi_{1}=2
$$

for all $x$. Furthermore,

$$
\phi^{\prime}(x)=\frac{-4 e^{x}}{\left(3+4 e^{x}\right)^{2}} \leq 0
$$

for all $x$. The paths of $\phi(x)$ and its derivative, $\phi^{\prime}(x)$, are shown in Figure 1

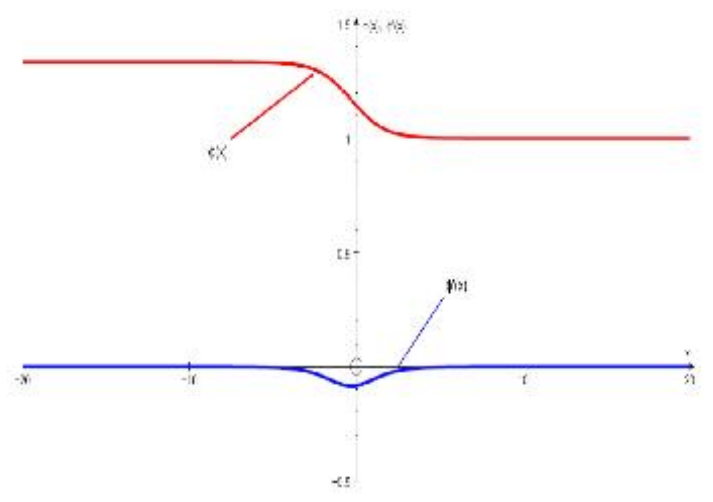

Figure 1. Functions $\phi(x(t))$, and $\phi^{\prime}(x(t))$

(ii) The function

$$
\begin{aligned}
& g(\cdot)=\frac{2+t^{2}+x^{2}(t-\tau(t))+y^{2}(t-\tau(t)) \phi^{-2}(x(t-\tau(t)))}{1+t^{2}+x^{2}(t-\tau(t))+y^{2}(t-\tau(t)) \phi^{-2}(x(t-\tau(t)))} \\
& =1+\frac{1}{1+t^{2}+x^{2}(t-\tau(t))+y^{2}(t-\tau(t)) \phi^{-2}(x(t-\tau(t)))}
\end{aligned}
$$


Since the fraction

$$
\frac{1}{1+t^{2}+x^{2}(t-\tau(t))+y^{2}(t-\tau(t)) \phi^{-2}(x(t-\tau(t)))} \geq 0
$$

for all $t \geq 0, x(t-\tau(t))$ and $y(t-\tau(t))$, it follows that

$$
g(\cdot) \geq a=1
$$

for all $t \geq 0, x(t-\tau(t))$ and $y(t-\tau(t))$.

(iii) The function

$$
h(x):=\frac{x\left(2+x^{2}\right)}{1+x^{2}}=x+\frac{x}{1+x^{2}}
$$

or

$$
\frac{h(x)}{x}=1+\frac{1}{1+x^{2}}
$$

Since

$$
0 \leq H(x)=\frac{1}{1+x^{2}} \leq 1
$$

for all $x$ it follows that

$$
1=b \leq \frac{h(x)}{x} \leq L=2
$$

for all $x \neq 0$.

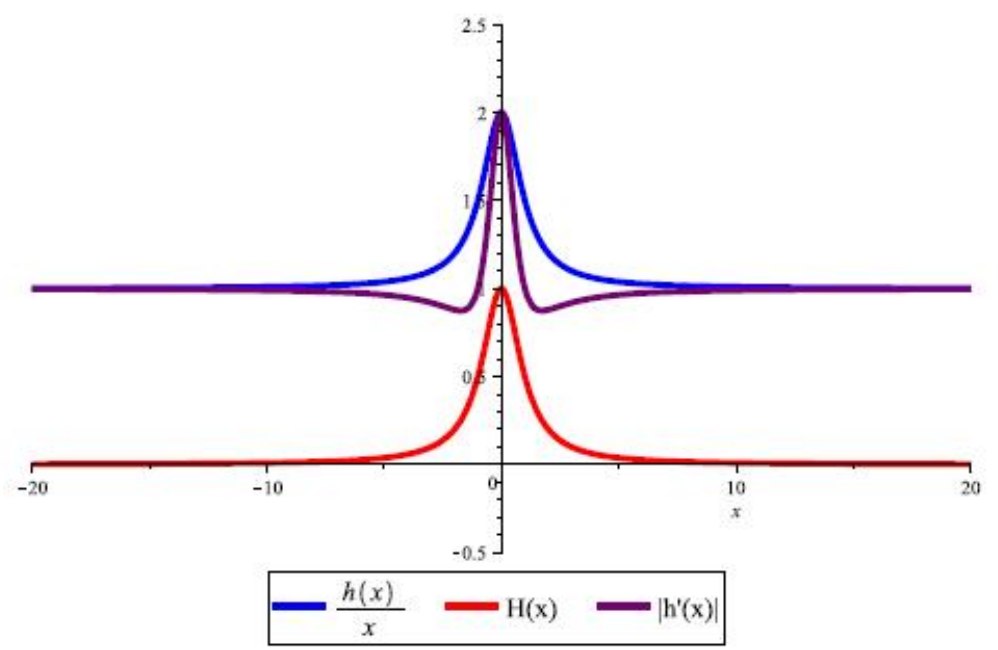

Figure 2. The behaviour of the functions $h(x) / x, H(x)$ and $\left|h^{\prime}(x)\right|$ 
Stability, Boundedness and periodic solutions to certain second... 273

Furthermore, the derivative of the function $h$ with respect to $x$ is

$$
h^{\prime}(x)=\frac{2+x^{2}+x^{4}}{\left(1+x^{2}\right)^{2}}=1+\frac{1-x^{2}}{\left(1+x^{2}\right)^{2}},
$$

Noting that

$$
\frac{1-x^{2}}{\left(1+x^{2}\right)^{2}} \leq 1
$$

for all $x$, it follows that

$$
h^{\prime}(x)=1+\frac{1-x^{2}}{\left(1+x^{2}\right)^{2}} \leq 2
$$

and

$$
\left|h^{\prime}(x)\right| \leq L=2
$$

for all $x$. The behaviour of the functions $h(x) / x, H(x)$ and $h^{\prime}(x)$ are shown in Figure 4

(iv) Using estimates (i) to (iii) of Example 4.1 with $\beta=\frac{1}{2}$, inequality (3.2) and equation (3.15) become

$$
\alpha<\min \left\{\frac{1}{2}, \frac{1}{22}\right\}=\frac{1}{22} \text { and } \lambda=32>0
$$

respectively.

(v) The function

$$
\begin{gathered}
p(\cdot)=\frac{2+t^{2}+\cos [x(t-\tau(t))]+\cos \left[y(t-\tau(t)) \phi^{-1}(x(t-\tau(t)))\right]}{2\left[t^{2}+\cos [x(t-\tau(t))]+\cos \left[y(t-\tau(t)) \phi^{-1}(x(t-\tau(t)))\right]\right]} \\
=\frac{1}{2}+\frac{1}{t^{2}+\cos [x(t-\tau(t))]+\cos \left[y(t-\tau(t)) \phi^{-1}(x(t-\tau(t)))\right]}
\end{gathered}
$$

It is not difficult to show that

$$
|p(\cdot)| \leq M=\frac{3}{2}
$$

for all $t \geq 0, x(t-\tau(t))$ and $y(t-\tau(t))$. 
From items (i) to (v) of Example 4.1, the assumption of Theorem 3.1, Theorem 3.6 and Theorem 3.7 hold, thus by Theorem 3.1, Theorem 3.6 and Theorem 3.7 the solution $\left(x_{t}, y_{t}\right)$ of system (4.2).

(i) is uniformly bounded and uniformly ultimately bounded;

(ii) possess a periodic solution of period $\omega$; and

(iii) is unique.

Also, if $p(\cdot)=0$ in system (4.2), items (i) to (iv) of Example 4.1 are equivalent to hypotheses (i) to (iv) of Theorem 3.5, then by Theorem 3.5 the trivial solution of system 4.2 is uniformly asymptotically stable.

Example 4.2. Consider also, the second order delay differential equation

$$
\begin{gathered}
{\left[\frac{5+6 e^{(2 x+1)}}{2+3 e^{(2 x+1)}} x^{\prime}\right]^{\prime}+\frac{3+\cos [t x(t-\tau(t))]+\cos \left[x^{\prime}(t-\tau(t))\right]}{2\left[1+\cos [t x(t-\tau(t))]+\cos \left[x^{\prime}(t-\tau(t))\right]\right]}} \\
+\frac{4 x(t-\tau(t))+7 x^{3}(t-\tau(t))+x(t-\tau(t)) \cos (2 x(t-\tau(t)))}{2+7 x^{2}(t-\tau(t))} \\
=\frac{3+4 t+2|x(t-\tau(t))|+2\left|x^{\prime}(t-\tau(t))\right|}{1+2 t+|x(t-\tau(t))|+\left|x^{\prime}(t-\tau(t))\right|}
\end{gathered}
$$

Equation (4.3) in its equivalent form is

$$
\begin{gathered}
x^{\prime}=\frac{2+3 e^{(2 x+1)}}{5+6 e^{(2 x+1)}} y \\
y^{\prime}=\frac{3+4 t+2|x(t-\tau(t))|+2\left|y(t-\tau(t)) \phi^{-1}(x(t-\tau(t)))\right|}{1+2 t+|x(t-\tau(t))|+\left|y(t-\tau(t)) \phi^{-1}(x(t-\tau(t)))\right|} \\
+\int_{t-\tau(t)}^{t} \frac{\left(7 x^{2}-2\right) \cos 2 x+2\left(7 x^{2}+2\right)\left(x \sin 2 x-7 x^{2}-2\right)}{\left(7 x^{2}+2\right)^{2}}(s) d s \\
-\frac{4 x+7 x^{3}+x \cos 2 x}{2+7 x^{2}} \\
-\frac{3+\cos [t x(t-\tau(t))]+\cos \left[y(t-\tau(t)) \phi^{-1}(x(1-\tau))\right]}{2\left[1+\cos [t x(1-\tau(t))]+\cos \left[y(t-\tau(t)) \phi^{-1}(x(t-\tau(t)))\right]\right]}
\end{gathered}
$$

Comparing system (1.2) with system (4.4), we find that 
Stability, Boundedness and periodic solutions to certain second... 275

(i) the function

$$
\phi(x):=\frac{5+6 e^{(2 x+1)}}{2+3 e^{(2 x+1)}}=2+\frac{1}{2+3 e^{(2 x+1)}} .
$$

Let

$$
\Phi(x):=\frac{1}{2+3 e^{(2 x+1)}} .
$$

It is not difficult to show that

$$
2=\phi_{0} \leq \phi(x) \leq \phi_{1}=3
$$

for all $x$. Moreover,

$$
\phi^{\prime}(x)=-\frac{6 e^{2 x+1}}{\left(2+3 e^{(2 x+1)}\right)^{2}} \leq 0
$$

for all $x$. The behaviour of the functions $\phi(x)$ and $\phi^{\prime}(x)$ are shown in Figure 3

(ii) the function

$$
\begin{array}{r}
g(\cdot):=\frac{3+\cos [t x(t-\tau(t))]+\cos \left[y(t-\tau(t)) \phi^{-1}(x(t-\tau(t))]\right.}{2\left[1+\cos [t x(t-\tau(t))]+\cos \left[y(t-\tau(t)) \phi^{-1}(x(t-\tau(t))]\right]\right.} \\
=\frac{1}{2}+\frac{1}{1+\cos [t x(t-\tau(t))]+\cos \left[y(t-\tau(t)) \phi^{-1}(x(t-\tau(t))]\right.}
\end{array}
$$

from where we obtain

$$
g(\cdot) \geq a=\frac{1}{2},
$$

for all $t \geq 0, x(t-\tau(t))$ and $y(t-\tau(t))$. 


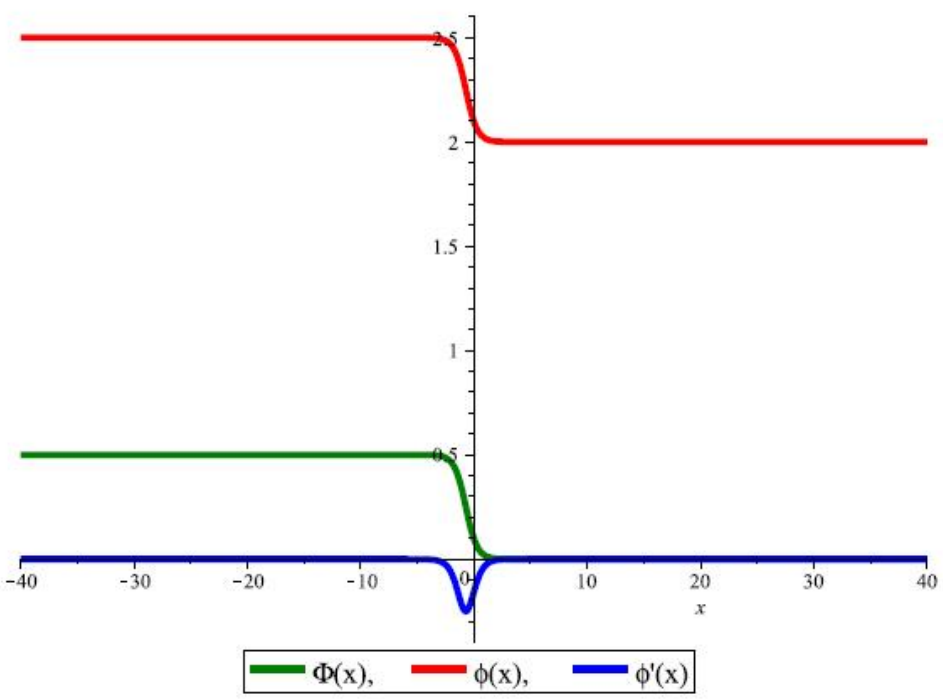

Figure 3. The behaviour of the functions $\Phi(x), \phi(x)$ and $\phi^{\prime}(x)$.

(iii) the function

$$
h(x):=\frac{4 x+7 x^{3}+x \cos 2 x}{2+7 x^{2}}=2 x+\frac{x \cos 2 x}{2+7 x^{2}} .
$$

This can be recast in the form

$$
\frac{h(x)}{x}=2+\frac{\cos 2 x}{2+7 x^{2}}=2+H(x),
$$

where

$$
H(x)=\frac{\cos 2 x}{2+7 x^{2}} .
$$

It is not difficult to show that

$$
-\frac{1}{2} \leq H(x) \leq \frac{1}{2}
$$

for all $x$. It follows that

$$
\frac{3}{2}=b \leq \frac{h(x)}{x} \leq L=\frac{5}{2}
$$

for all $x \neq 0$. In addition,

$$
\left|h^{\prime}(x)\right| \leq L=\frac{5}{2}
$$


Stability, Boundedness and periodic solutions to certain second... 277

for all $x$. Alternatively,

$$
\begin{aligned}
h^{\prime}(x) & =\frac{\left(7 x^{2}-2\right) \cos 2 x+2\left(7 x^{2}+2\right)\left(x \sin 2 x-7 x^{2}-2\right)}{\left(7 x^{2}+2\right)^{2}} \\
& =2-\frac{\left(7 x^{2}-2\right) \cos 2 x+2 x\left(7 x^{2}+2\right) x \sin 2 x}{\left(7 x^{2}+2\right)^{2}}
\end{aligned}
$$

It follows that

$$
\left|h^{\prime}(x)\right| \leq L=\frac{5}{2}
$$

for all $x$. The behaviour of the functions $h(x) / x, H(x)$ and $\left|h^{\prime}(x)\right|$ are shown in Figure 3
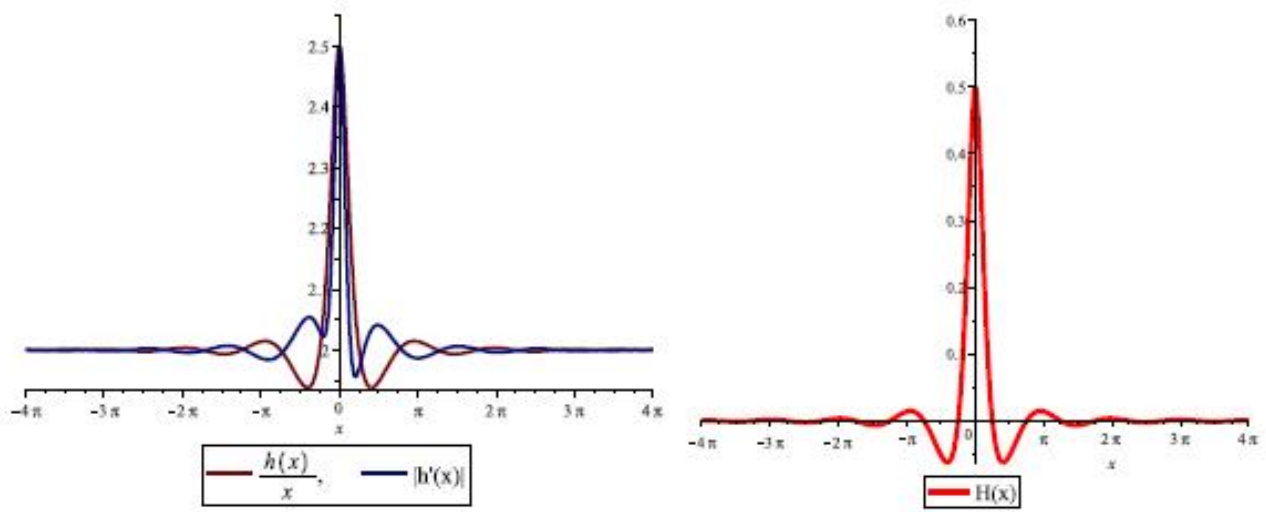

Figure 4. The behaviour of the functions $h(x) / x, H(x)$ and $\left|h^{\prime}(x)\right|$.

(iv) From items (i) - (iii) of Example 4.2, choose $\beta=\frac{1}{2}$ inequality 3.2 and equation (3.15) become

$$
\alpha<\min \left\{\frac{6}{5}, \frac{6}{175}\right\}=\frac{6}{175}, \text { and } \lambda=270>0
$$

respectively.

(v) Finally, the function

$$
p(\cdot):=\frac{3+4 t+2|x(t-\tau(t))|+2\left|y(t-\tau(t)) \phi^{-1}(x(t-\tau(t)))\right|}{1+2 t+|x(t-\tau(t))|+\left|y(t-\tau(t)) \phi^{-1}(x(t-\tau(t)))\right|}
$$




$$
=2+\frac{1}{1+2 t+|x(t-\tau(t))|+\left|y(t-\tau(t)) \phi^{-1}(x(t-\tau(t)))\right|}
$$

where we obtain

$$
|p(\cdot)| \leq M=3
$$

for all $t \geq 0, x(t-\tau(t))$ and $y(t-\tau(t))$.

From items (i) to (v) of Example ??, assumptions of Theorem 3.1, Theorem 3.6 and Theorem 3.7 hold, thus by Theorem 3.1, Theorem 3.6 and Theorem 3.7 the solution $\left(x_{t}, y_{t}\right)$ of system (4.4)

(i) is uniformly bounded and uniformly ultimately bounded;

(ii) possess a periodic solution of period $\omega$; and

(iii) is unique.

Also, if $p(\cdot)=0$ in system (4.4), items (i) to (iv) of Example 4.2 satisfy the assumptions of Theorem 3.5, then by Theorem 3.5 the trivial solution of system 4.4 is uniformly asymptotically stable.

\section{Acknowledgments}

The authors are grateful to the anonymous referee(s) and the handling editor for their valuable comments and useful suggestions that have improved the quality of this work.

\section{References}

[1] Ademola, A. T. Boundedness and stability of solutions to certain second order differential equations. Differential Equations and Control Processes N 3, Volume 2015.

[2] Ademola, A. T., Moyo, S., Ogundare, B. S., Ogundiran, M. O. and Adesina, O. A. Stability and boundedness of solutions to a certain second order non autonomous stochastic differential equation, International Journal of Analysis, Volume 2016, (2016), Article ID 2843709, 10 pages, doi.org/10.1155/2016/2843709. 
[3] Ademola, A. T., Ogundare, B.S., Ogundiran, M. O. and Adesina, O. A. Periodicity, stability, and boundedness of solutions to certain second order delay differential equations International Journal of Differential Equations Volume 2016, (2016), Article ID 2843709, 10 pages, doi.org/10.1155/2016/2843709.

[4] Alaba, J. G. and Ogundare, B. S. On stability and boundedness properties of solutions of certain second order non-autonomous nonlinear ordinary differential equation, Kragujevac Journal of Mathematics Volume 39 (2), pp. 255 - 266, (2015).

[5] Burton, T. A. Stability and periodic solutions of ordinary and functional differential equations. Mathematics in Science and Engineering, 178 Academic Press. Inc., Orlando, FL, (1985).

[6] Burton, T. A. Volterra integral and differential equations. New York: Academic Press, (1983).

[7] Cahlon, B. and Schmidt, D. Stability criteria for certain second order delay differential equations with mixed coefficients, J. Comput. Appl. Math. 170, pp. 79 - 102, (2004).

[8] Driver, R. D. Ordinary and delay differential equations, SpringerVerlag New York Heidelberg Berlin, (1977).

[9] Domoshnitsky, A. Non oscillation, maximum principles, and exponential stability of second order delay differential equations without damping term. Domoshnitsky Journal of Inequalities and Applications 2014, (2014):361.

[10] Grigoryan, G. A.; Boundedness and stability criteria for linear ordinary differential equations of the second order, Russian Mathematics (Iz. VUZ), Vol. 57, No. 12, pp. 8 - 15, (2013).

[11] Hale, J. K. Theory of functional differential equations. Springer-Verlag New York, (1977).

[12] Jin, Z., and Zengrong, L. On the global asymptotic behaviour of solutions to a non autonomous generalized Lienard system. J. Math. Res. Expo. 21 No.3, pp. 410 - 414, (2001).

[13] Kolmanovskii, V., and Myshkis, A. Applied theory of functional differential equations, Springer-Science+Business Media, B. V., (1992). 
[14] Kroopnick A. J. Bounded solutions to $x^{\prime \prime}+q(t) b(x)=f(t)$, Int. J. Math. Edu. Sci. Tec. 41, 6, pp. 829 - 836, (2010).

[15] Kuag, Y. Delay differential equations with applications in populations dynamics, Mathematics in Science and Engineering, 191 Academic Press. Inc., Orlando, FL, (1985).

[16] Lakshmikantham, V., Wen, L., and Zhang, B. Theory of differential equations with unbounded delay, Springer Science+Business Media Dordrecht, (1994).

[17] Ogundare, B. S., Ademola, A. T., Ogundiran, M. O. and Adesina, O.A. On the qualitative behaviour of solutions to certain second order nonlinear differential equation with delay. Ann Univ Ferrara,, pp. 1 21, (2016).

[18] Ogundare, B. S. and Afuwape, A. U. Boundedness and stability properties of solutions of generalized Lienard equation, Kochi J. Math., 9, pp. 97 - 108, (2014).

[19] Ogundare, B. S. and Okecha, G. E. Boundedness, periodicity and stability of solution $\ddot{x}+a(t) g(\dot{x})+b(t) h(x)=p(t ; x, \dot{x})$, Math. Sci. Res. J., Vol. 11 No. 5, pp. 432 - 443, (2007).

[20] Tunç, C. A note on the bounded solutions to $x^{\prime \prime}+c\left(t, x, x^{\prime}\right)+q(t) b(x)=$ $f(t)$, Appl. Math. Inf. Sci., Vol. 8, No. 1, pp. 393 - 399, (2014).

[21] Tunç, C. Boundedness analysis for certain two-dimensional differential systems via a Lyapunov approach, Bull. Math. Soc. Sci. Math. Roumanie (N. S.) 53, (101), pp. 61 - 68, (2010).

[22] Tunç, C. New Results on the existence of periodic solutions for Rayleigh equation with state-dependent delay. J. Math. Fund. Sci., Vol. 45, No. 2, pp. 154 - 162, (2013).

[23] Tunç, C. Stability and boundedness in multi delay vector Liénard equation. Filomat 27 : 3, (2013), 435 - 445, DOI 10.2298/FIL1303435T.

[24] Tunç, C. Stability and boundedness of solutions of non-autonomous differential equations of second order, J Comput. Anal. Appl. 13, No. 6, pp. 1067 - 1074, (2011). 
[25] Tunç, C. Uniformly stability and boundedness of solutions of second order nonlinear delay differential equations. Appl. Comput. Math. 10, No. 3, pp. 449 - 462, (2011).

[26] Wang, F. and Zhu, H. Existence, uniqueness and stability of periodic solutions of a duffing equation under periodic and anti-periodic eigenvalues conditions. Taiwanese Journal of Mathematics Vol. 19, No. 5, pp. 1457 - 1468, October (2015) DOI:10.11650/tjm.19.2015.3992

[27] Xu, An Shi. Boundedness and stability of solutions to second-order delay and non delay differential equations. (Chinese) Chinese Ann. Math. Ser. A 9, No. 5, 615-622, (1988).

[28] Yeniçerioğlu, A. F. The behavior of solutions of second order delay differential equations. J. Math. Anal. Appl. 332, pp. 1278 - 1290, (2007).

[29] Yeniçerioğlu, A. F. Stability properties of second order delay integrodifferential equations. Computers and Mathematics with Applications 56, pp. 3109-3117, (2008).

[30] Yoshizawa, T. Liapunov's function and boundedness of solutions, Funkcial. Ekvac. 2, pp. 95-142, (1959).

[31] Yoshizawa, T. Stability theory and existence of periodic solutions and almost periodic solutions, Spriger-Verlag, New York. Heidelberg. Berlin, (1975).

[32] Yoshizawa, T. Stability theory by Liapunov's second method, The Mathematical Society of Japan, (1966).

[33] Zhu, Y. F. On stability, boundedness and existence of periodic solution of a kind of third order nonlinear delay differential system, Annals of Diff. Equations Vol. 8, No. 2, pp. 249-259, (1992).

\author{
A. T. Ademola \\ Department of Mathematics, \\ Obafemi Awolowo University, \\ Ile-Ife, \\ Nigeria \\ e-mail : atademola@oauife.edu.ng
}




\section{P. O. Arawomo}

Department of Mathematics, University of Ibadan, Ibadan,

Nigeria

e-mail : womopeter@gmail.com

and

\section{A. S. Idowu}

Department of Mathematics, University of Ilorin,

Ilorin,

Nigeria

e-mail : asidowu@gmail.com 